\title{
REVIEW OF CANCER STATISTICS IN INDIA
}

\author{
Priya D, \\ Department of Electronics and Communication Engineering, \\ Kings College of Engineering, \\ Tanjore, India \\ priyaramsaro@gmail.com \\ Muthulekshmi M, \\ Department of Electronics and Communication Engineering, \\ Vivekanandha College of Engineering For Women, \\ Tiruchengode, Namakkal, India \\ srimanlekswar@gmail.com
}

\begin{abstract}
This study reports key information's about breast and cervical cancer in India. Among various health-related issues, the maximum mortality is due to cancers of different types. Due to the late diagnosis and treatment in India, 0.3 millions of people died per year and increasingly every year. According to diverse cancers breast and cervix carcinomas are considered as the deadliest carcinoma due to its increased mortality among Indian women. As per cancer statistics in 2012, 4.77 lakh and 5.37 lakh men and women are affected by different types of cancers in India. This review focuses the Indian statistics of breast cancer along with cervical cancer.
\end{abstract}

Keywords: Breast Cancer, Cervical Cancer, Mortality, Incidence

\section{INTRODUCTION}

In 2012, 32.6 million people lived with carcinoma in the world. They were diagnosed within five years of interval. Also, 8.2 million people died in the same year due to cancers only. The number of new cases was 14.1 million in 2012 . Among these statistics, the occurrence of cancer cases, cancer deaths, and new cancer cases in the less developed regions were $48 \%, 65 \%$ and $57 \%$ respectively.

The cancer incidence rate (age-standardized) in men was 205 per 100000. In women, it was around 165 per 100000 . The highest rate of incidence in men was found in Australia/New Zealand It was around 365 per 100000. The lowest rate of incidence in men was only 79 per 100000 in Western Africa. In women, the lowest rate (103 per 100000) was found in South-Central Asia, and highest rate (295 per 100000) was found in Northern America.

The mortality rate of the developed region is higher than the less developed region. It was $15 \%$ higher in men and $8 \%$ higher in women. The highest rate of mortality in women was found in Melanesia and Western Africa. It was around 119 per 100000 in Melanesia and 111 per 100000 in Western Africa. The lowest rate of mortality in women was 72 per 100000 in Central America and 65 per 100000 in South Central Asia [1]. In men, the lowest rate (69 per 100000) was found in Western Africa, and highest rate (173 per 100000) was found in Central and Eastern Europe. 


\section{CANCER SCENARIO IN INDIA}

\section{A. Breast Cancer}

It is the most diagnosed carcinoma of malignant in the world and has the highest mortality rate in India. The Indian Council of Medical Research (ICMR) conducted surveys about cancer from 1982 to 2005 in the following metro cities; Chennai, Delhi, Bangalore and Mumbai. From the survey, it is observed that the number of new breast cancer cases is around 100000 every year and increases rapidly. The word mortality refers to the number of deaths due to cancer and incidence refers to the number of new cases. Cancer statistics are analyzed using Globocan data for the year 2008 and 2012. Figure 1 and 2 show the Cancer incidence (Breast cancer vs other cancers) in India for the year 2008 and 2012 respectively.

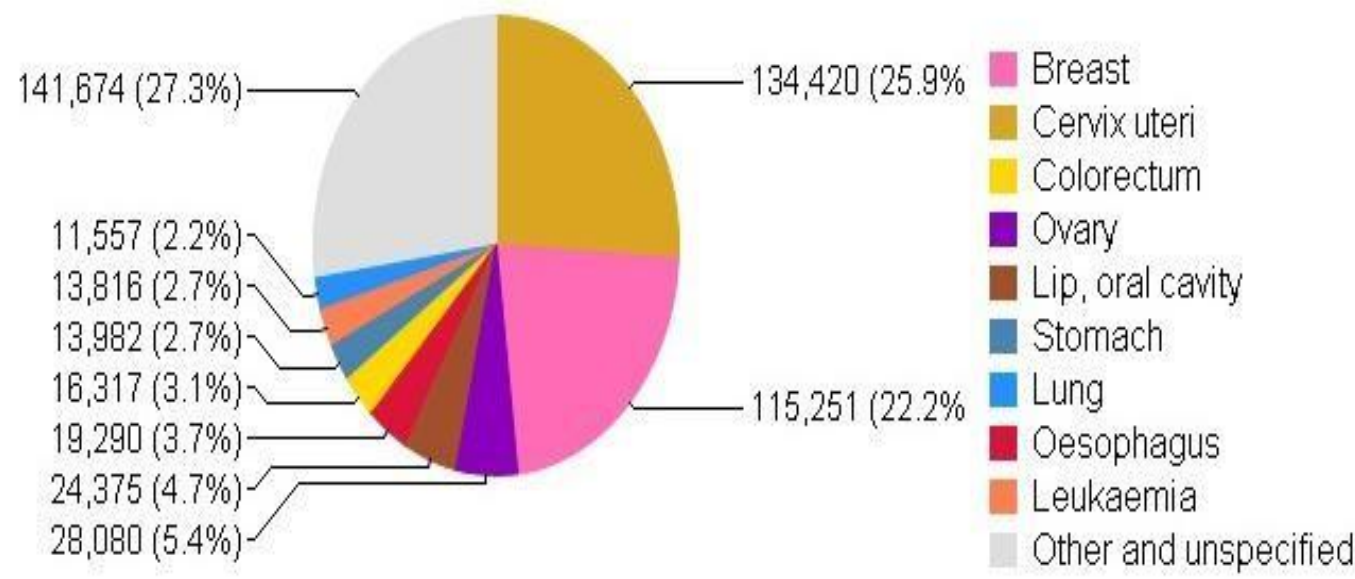

5DURCE; HTTP://ELDBUCFN.IFRE.FR

Fig. 1 Cancer incidence (Breast cancer vs other cancers) in India (Estimations from Globocan 2008)

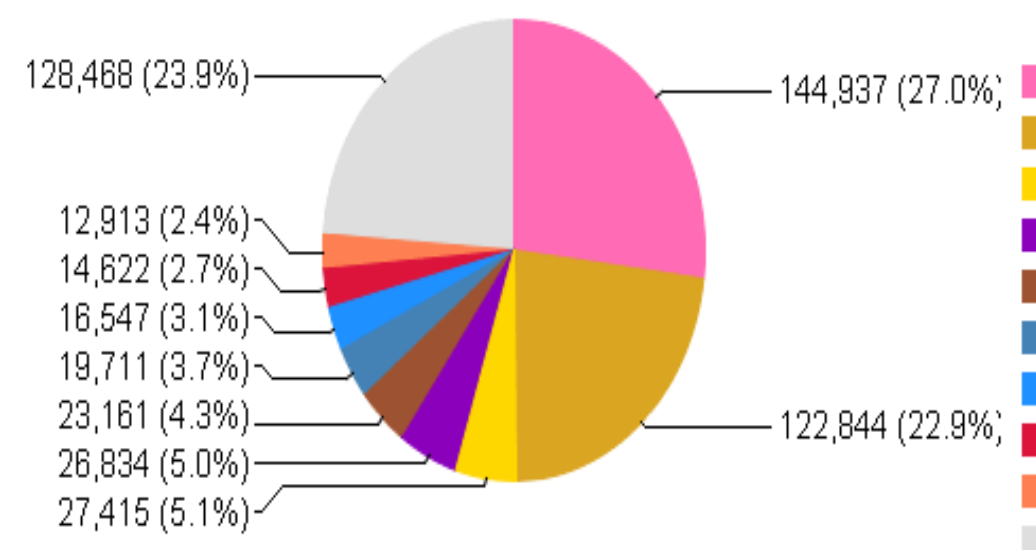

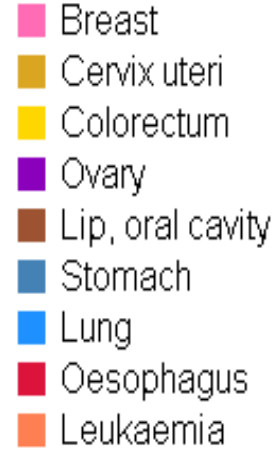

Other and unspecified 
It is evident from the Figures 1 and 2 that the incidence of breast cancer from 2008 to 2012 is increased around $1.1 \%$. Among the number of cancer patients, $27 \%$ were diagnosed with breast cancer. After breast cancer, the leading cancer incidence is cervical cancer, nearly $22.90 \%$ Indian people were diagnosed with cervical cancer in 2012 [3].

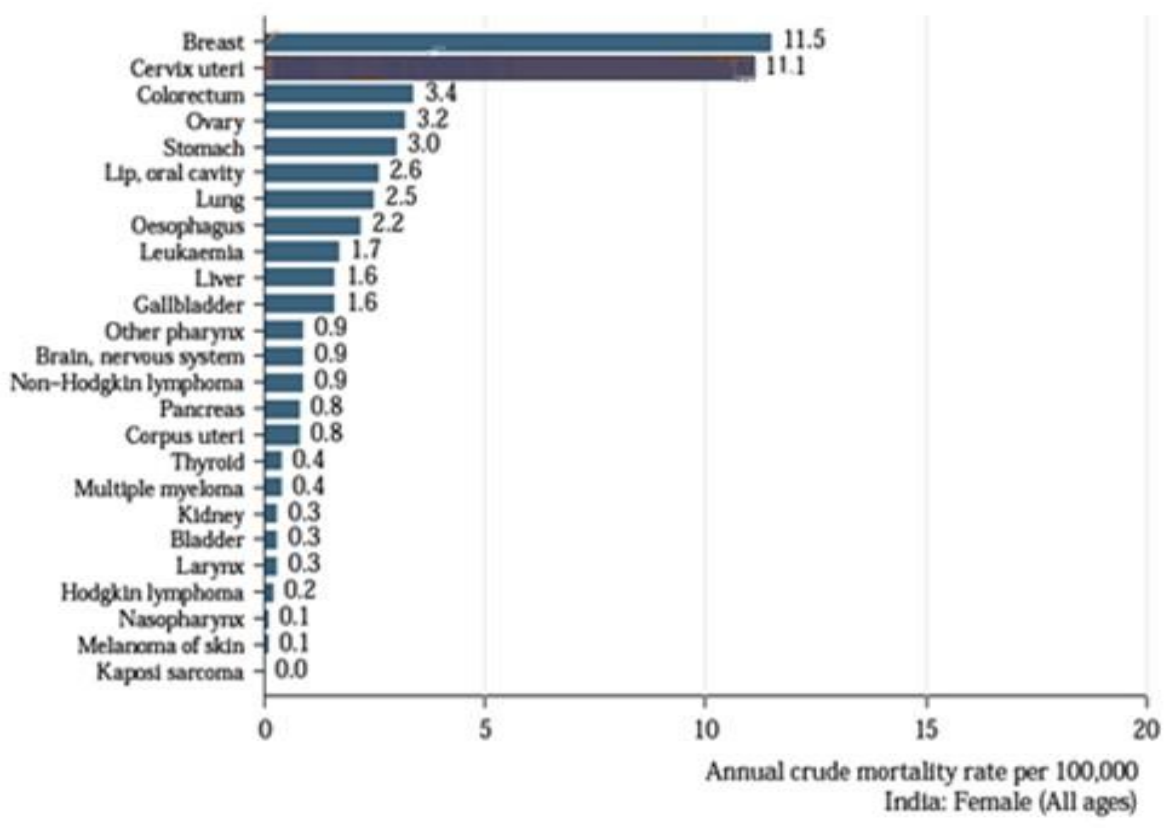

Fig. 3 Mortality rate (Cervical cancer vs other cancers) in India (2012 estimation)

\section{A. Cervical Cancer}

In India, the incidence of cervical cancer increases recently. However, the incidence of breast cancer increases steadily over the last ten years. It is noted that the occurrence of breast cancer is higher than cervical cancer in India based on 2012 estimation. The mortality rate of breast cancer is also higher than cervical cancer. Based on the 2012 estimation, annually 67477 deaths occur due to cervical cancer, and it is the 2 nd cause of deaths among women in India. In India, women aged about 15 to 44 years are mostly affected by cervical cancer [1]. The mortality rate of cervical cancer is shown in Figure 4.

TABLE 1 Cervical cancer mortality in India (2012estimations)

\begin{tabular}{|c|c|}
\hline Indicator & India \\
\hline Annual number of deaths & 67,477 \\
\hline Crude mortality rate & 11.1 \\
\hline Age-standardized mortality rate & 12.4 \\
\hline Cumulative risk (\%) at 75 years old & 1.4 \\
\hline
\end{tabular}




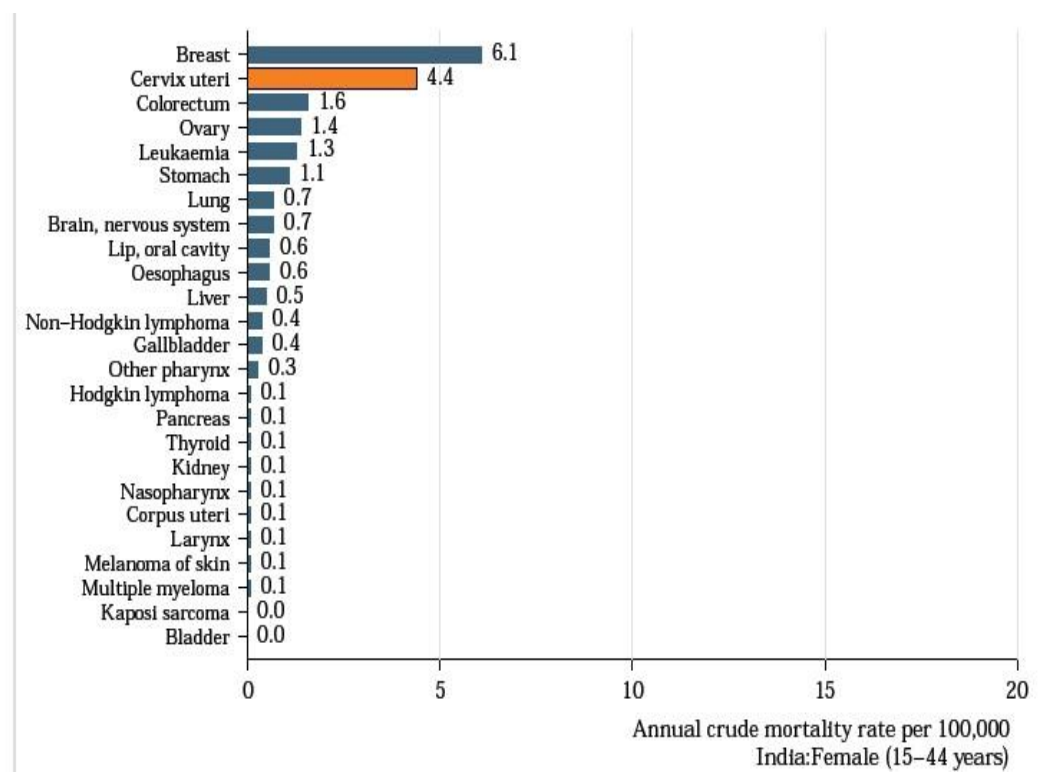

Fig. 4 Age-specific mortality rates for women from 2012 estimations (Cervical cancers vs other cancers)

\section{CONCLUSION}

Breast cancer tends to lead high mortality among women in India. The only way to prevent breast related death, the automated early diagnosis is much needed. Although digital mammography is the accepted imaging modality for breast cancer screening, interpreting mammogram images is complicated with lower sensitivity and specificity. Hence, to provide radiologists with a visual prognosis automated computer-aided diagnosis is essential for accurate breast cancer diagnosis in future. It is a technology used for the detection of cancer by its characteristics and should be designed for all type of cancers in the world.

\section{REFERENCES}

[1]. Human Papilloma viruses and Related Diseases Report, ICO HPV Information Centre, pp.1-69.

[2]. http://globocan.iarc.fr/Pages/fact_sheets_cancer.aspx

[3]. http://www.breastcancerindia.net/bc/statistics/stat_global.htm. 